\title{
L'empreinte symbolique des grenouilles et crapauds dans la culture Mochica du Pérou : de l'analyse iconographique à la portée sociale
}

\section{Sandra Giuliato}

\author{
(2) OpenEdition \\ Journals \\ Édition électronique \\ URL : http://journals.openedition.org/elohi/503 \\ DOI : 10.4000/elohi.503 \\ ISSN : 2268-5243 \\ Éditeur \\ Presses universitaires de Bordeaux
}

\section{Édition imprimée}

Date de publication : 1 janvier 2015

Pagination : 121-145

ISBN : 979-10-300-0044-3

ISSN : 2431-8175

Référence électronique

Sandra Giuliato, «L'empreinte symbolique des grenouilles et crapauds dans la culture Mochica du Pérou : de l'analyse iconographique à la portée sociale », ELOHI [En ligne], 7 | 2015, mis en ligne le 01 janvier 2016, consulté le 10 décembre 2020. URL : http://journals.openedition.org/elohi/503 ; DOI : https://doi.org/10.4000/elohi.503 


\section{L'empreinte symbolique des grenouilles et crapauds dans la culture Mochica du Pérou : de l'analyse iconographique à la portée sociale}

\section{SANDRA GIULIATO}

UMR 5060 IRAMAT, Université Bordeaux Montaigne

\section{Introduction}

Dans le Pérou ancien, grenouilles et crapauds ont été le sujet récurrent de représentations spécifiques reflétant la valeur accordée à l'animal au sein de systèmes de pensée culturellement définis (Larco Hoyle : 105 ; Lavallée : 140-141; Hocquenghem : 252-253; McClelland).

Le choix d'analyser les réalisations dépeignant ces animaux dans la société Mochica tient à plusieurs raisons : l'existence d'une recherche archéologique dynamique amenant à l'acquisition croissante de données de terrain (Quilter : 151152 ; Castillo Butters et al. : 14) ; une localisation spatiale en contexte de vallées qui ont été le siège d'adaptations locales à des valeurs culturelles communément partagées (Millaire : 6186; voir ici-même, 1.1) ; une occupation chrono-culturelle étendue qui a exprimé différentes phases de développement régional; et, enfin, une trame chronologique à la croisée de cultures locales, autorisant une mise en perspective des représentations picturales mochicas avec celles exprimées par d'autres peuples de l'aire andine (Stone-Miller : 8).

Cependant, saisir les interactions culturelles qui interviennent entre les groupes humains et leur environnement nécessite un savoir empirique qui ne nous est pas accessible dans le cas des peuples disparus. L'empreinte symbolique, autrement dit la portée sociale du thème perçue au travers des expressions matérielles, exige, de fait, une réflexion basée sur les vestiges en tant que témoins directs, mais également le recours à une nécessaire interdisciplinarité permettant de croiser regards et connaissances. C'est ainsi que l'histoire de l'art, l'archéologie, 
l'ethnologie et l'herpétologie ${ }^{1}$ ont participé à la compréhension de l'empreinte sociale des amphibiens anoures ${ }^{2}$ au sein de la culture Mochica (Lavallée : 27 ; Venegas ; Goepfert : 431-444 ; Golte : 169-174). Par le biais des témoignages matériels se dessinent, ainsi, les rapports que les Mochicas ont tissés avec l'animal, lesquels autorisent la formulation de plusieurs hypothèses quant aux spécificités et aux évolutions formelles d'un discours iconographique qui donne à voir une partie de la pensée sociale et mythique d'un peuple. A cet effet, il nous faut tout d'abord appréhender les spécificités de l'environnement auquel était soumis le peuple Mochica, ainsi que les caractéristiques culturelles de cette civilisation, afin de déterminer les implications sociales potentiellement véhiculées par la représentation des grenouilles et crapauds. La diversité des figurations nous amène à envisager une portée symbolique impliquant les pluies et l'agriculture ainsi que les échanges entre mondes visible et invisible, et à prendre en considération la mutation probable de ces liens identifiés.
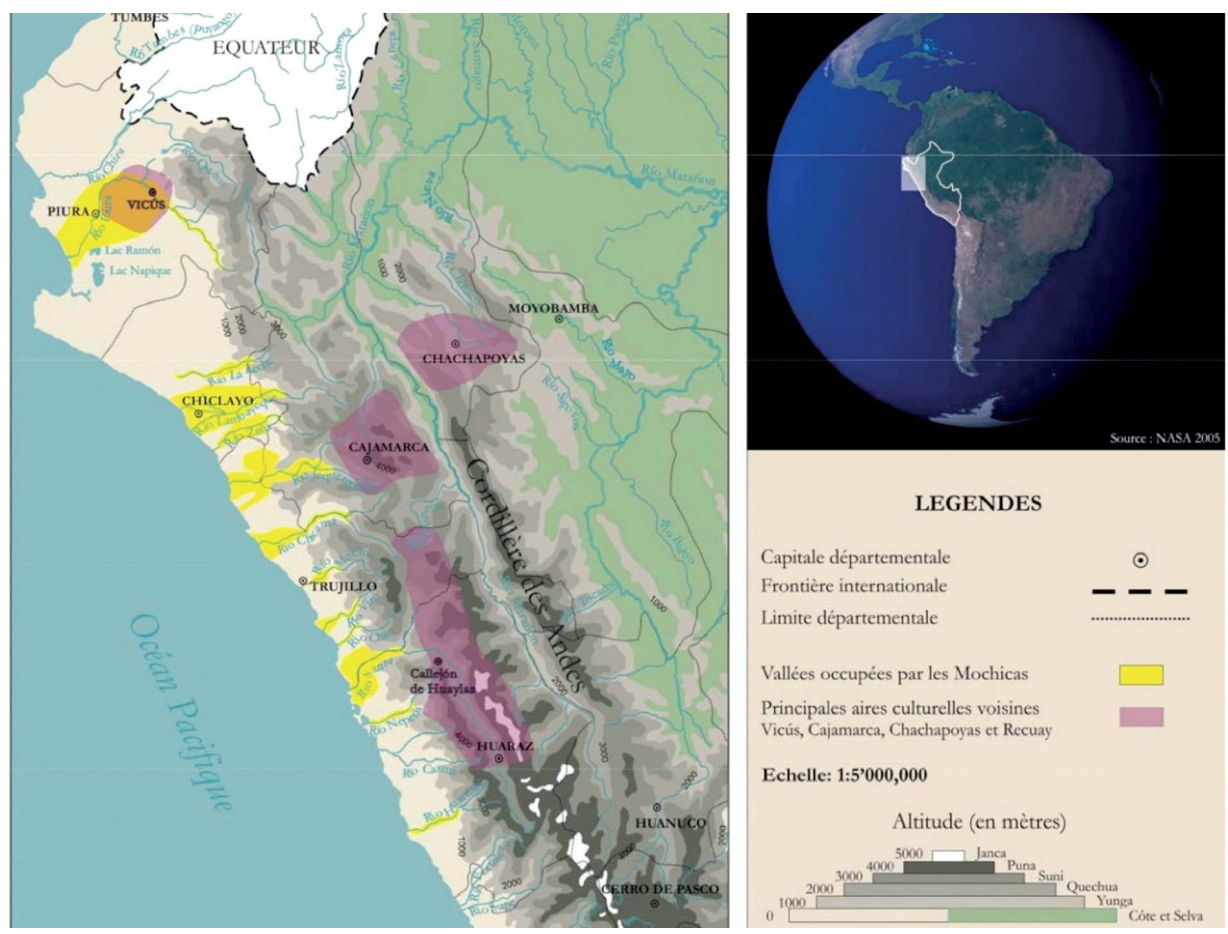

LEGENDES

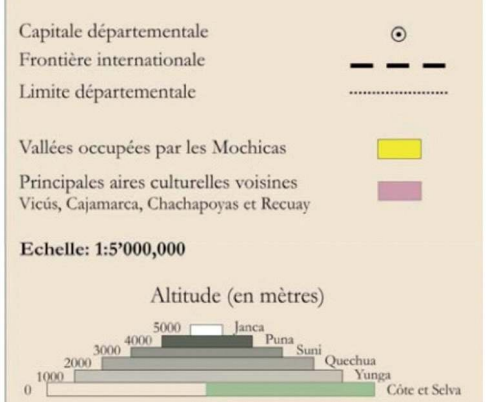

Figure 1 - Géographie de la région septentrionale du Pérou, indiquant les zones naturelles et leurs altitudes, ainsi que les aires culturelles mochicas et voisines.

Source : Fraresso 2007 : 14, fig. 1, d'après Peñaherrera del Aguila 1989.

1. Science qui étudie les amphibiens et les reptiles. Voir Parsons ; O’Shea et Halliday; Santiani.

2. Animaux qui se caractérisent par l'absence de queue à l'âge adulte (Santiani : 46). 


\section{Société mochica, trame environnementale et figuration des anoures}

Le peuple Mochica a occupé la côte septentrionale du Pérou, depuis la vallée de Piura, au nord, jusqu'à la vallée de Huarmey, au sud, sur une période comprise entre les $\mathrm{I}^{\mathrm{er}}$ et $\mathrm{IX}^{\mathrm{e}}$ siècle apr. J.-C. ${ }^{3}$

Les conditions climatiques qui caractérisent l'environnement immédiat des Mochicas interviennent de manière manifeste dans leur relation aux amphibiens anoures et dans des modalités de figuration à la portée symbolique complexe.

\section{Une société agricole aux prises avec un environnement incommode}

Plusieurs hypothèses sont émises sur l'origine et l'organisation de la société mochica, depuis l'établissement de quelques cités contrôlant de vastes territoires voisins jusqu'à la présence d'une myriade d'entités politiques éparpillées le long du littoral (Millaire : 6186). Dans les deux cas, la cohésion culturelle aurait été maintenue par un réseau complexe de relations existant entre - ainsi qu'à l'intérieur même - des vallées côtières dont les caractéristiques naturelles ont appelé à des adaptations spécifiques en fonction des zones de peuplement (Fraresso : 20-27).

Depuis les années 1990, la recherche archéologique a permis de déceler deux aires culturelles distinctes - Mochicas du Nord et Mochicas du Sud - séparées géographiquement par le désert de Paiján (Quilter : 160). Profondément marquée par son cadre environnemental, la société mochica a dû s'adapter aux conditions climatiques de l'étroite bande littorale qui caractérise sa zone de peuplement, enserrée à l'est par les Andes et à l'ouest par l'océan Pacifique. Cette région se compose d'un désert à la végétation éparse entrecoupé de vallées, lieux d'écoulement de rivières descendant le versant occidental du massif andin et permettant l'apport hydrique nécessaire à la création d'espaces fertiles (Dillehay et Kolata : 4326; Goepfert : 30). Les Mochicas y avaient basé leur économie sur la domestication des camélidés - en particulier l'élevage de lamas (Quilter : 156) -, l'exploitation des ressources du littoral - notamment la pêche et le commerce de spondyles (Ibid: 157) - et une agriculture à grande échelle (cultures de maïs, coton, haricots, etc.) facilitée par une irrigation efficace (Hill). Soumis à des changements à la périodicité et à la fréquence incertaines, le climat de la côte nord du Pérou a contraint ce peuple à adopter diverses stratégies pour faire face à des phénomènes violents tels que sécheresses persistantes (Dillehay et Kolata : 4325), fortes variations du débit des rivières, ou encore phénomène climatique d'El Niño entraînant inondations et destructions des habitats humains et naturels (Quilter : 155).

3. Pour information générale, nous renvoyons le lecteur aux ouvrages de Castillo Butters et al., Quilter, Bourget et Jones, Koon et Alex. 
L'environnement naturel rejaillit sur les productions iconographiques qui sont à envisager selon une double perspective de figuration réaliste et de pensée analogiste ${ }^{4}$, l'une et l'autre intervenant à des degrés divers. Car les artisans représentaient la nature au travers des relations que la société entretenait avec elle, faites de consensus et d'équilibre fragile; à ce titre, les animaux participaient pleinement au discours religieux - mythologique et rituel - en faisant figure d'acteurs principaux dans les activités cérémonielles (Franco et Vilela : 129). Entamée à partir du $\mathrm{VI}^{\mathrm{e}}$ siècle, la disparition de la culture Mochica s'est faite de manière plus ou moins abrupte d'une vallée à l'autre, ce qui explique la disparité des évolutions affectant les traditions sociales et les productions picturales (Koon et Alex : 1052).

\section{Les amphibiens anoures et la figuration mochica : nature et limite de l'étude}

Une collecte systématique d'objets représentant des amphibiens anoures nous a permis de constituer un corpus de référence de 461 pièces (Giuliato : 80) composé à $96 \%$ d'éléments céramiques 5 .

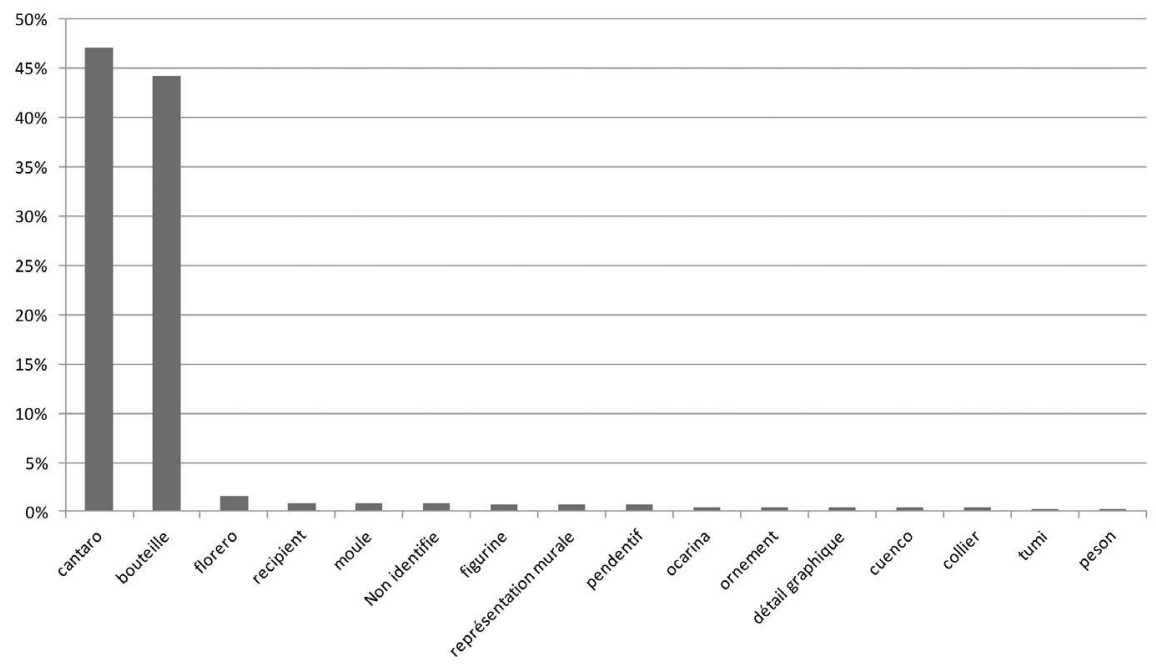

Figure 2 - Variabilité des types de support (tous matériaux) utilisés pour la représentation des amphibiens anoures dans la culture Mochica.

4. Ce mode de pensée considère la figuration comme une restitution visuelle des liens enchevêtrés supposés exister entre le sujet et le vaste ensemble d'agrégats dynamiques qui constitue le monde (Descola : 10).

5. Les termes en langue espagnole, tels que cántaro, florero et cuenco, font partie de la nomenclature utilisée en archéologie péruvienne pour caractériser des formes céramiques (cela étant, leur sens commun est celui de cruche, vase et terrine; note des éditeurs). Par ailleurs, le tumi désigne un couteau cérémoniel (Fraresso : 269). 
Ces derniers proviennent, pour une grande part, des collections du musée Larco de Lima, issues principalement de sites funéraires de la zone Mochica Sud 6 .

Les conditions de conservation de la terre cuite jouent un rôle indéniable dans sa transmission, à l'inverse des matières organiques plus facilement dégradables ou des métaux retransformés. Pour autant, les quantités rencontrées témoignent en faveur d'un mode d'expression privilégié. Afin d'étudier les spécificités et évolutions de ces représentations dans le temps et l'espace, la typochronologie céramique établie en 1946 par l'archéologue Rafael Larco Hoyle nous a servi de référence. Ce système chronologique de datation relative propose une sériation selon cinq phases céramiques (Mochica I à V), basées sur les changements survenus dans la forme du col des bouteilles à anse goulot étrier (Pillsbury). Ultérieurement, une séquence spécifique à la zone Mochica Nord a été réalisée en 1994, par Luis Jaime Castillo et Christopher Donnan, de manière à proposer une typologie plus caractéristique du développement local, selon les phases « Mochica Initial », « Moyen » et « Tardif » (Fraresso : 42).

Plusieurs éléments doivent cependant être signalés en ce qui concerne les limites propres à l'interprétation iconographique. Tout d'abord, la faible proportion d'objets bénéficiant d'une contextualisation précise $^{7}$ nous prive de données utiles concernant l'emplacement du site, l'organisation de l'espace, etc. Ensuite, l'analyse visuelle des artefacts via l'iconographie s'avère génératrice d'opinions parfois contradictoires ${ }^{8}$; car, si la mise en forme de la matière sert à véhiculer une idée ${ }^{9}$, alors les variations observables sur les productions matérielles mochicas dénotent un choix délibéré d'accentuation de traits propres à atteindre des objectifs symboliques précis - choix qui limite l'objectivité du raisonnement posé a posteriori. Des travaux anthropologiques montrent, par ailleurs, les erreurs qui peuvent résulter d'une étude visuelle déconnectée du terrain humain qui est à l'origine de l'image (Karadimas), d'où notre prudence quant à la formulation de certaines hypothèses qui ouvrent néanmoins le champ des possibles.

6. Les recherches documentaires ayant permis le recensement des pièces du corpus ont été complétées par plusieurs visites de terrain (collections muséales, sites archéologiques, etc.) comprenant deux déplacements au Pérou en 2009 et 2011.

7. Celle-ci est bien souvent absente, notamment lorsqu'il s'agit de découvertes anciennes ou clandestines.

8. Dès 1977, Anne-Marie Hocquenghem signale que les interprétations sont intimement liées aux objectifs des iconologues, dont les hypothèses et affirmations s'établissent autant en fonction de leur méthodologie que de leur personnalité (Hocqueghem : 7).

9. Au-delà de sa valeur décorative, l'objet renferme souvent une valeur narrative et/ou symbolique mise en évidence par le biais de l'analyse iconographique et de l'étude du contexte archéologique. Voir Goepfert : 155-168; de même, Giuliato : 103-104. 
La littérature sur les représentations d'amphibiens anoures précolombiens témoigne de fréquentes divergences d'interprétation ${ }^{10}$, à commencer par l'attribution arbitraire des termes grenouille ou crapaud ${ }^{11}$, espèces qui se distinguent pourtant par différents traits. Les grenouilles recherchent, en effet, la proximité de points d'eau, sont pourvues de pattes postérieures faites pour sauter et possèdent une peau relativement lisse, à l'inverse de celle, souvent verruqueuse, du crapaud. Animal majoritairement terrestre, ce dernier privilégie en revanche la marche et se révèle bien adapté aux conditions arides (Santiani : 15 et 47). Notons que, parmi les 480 espèces d'amphibiens anoures présentes au Pérou, seules quelques-unes sont proprement endémiques à la zone côtière septentrionale (Venegas). Les espèces de crapauds rencontrées sur le territoire mochica comprennent Bufo marinus, Bufo spinulosus et Bufo spinulosus limensis. Quant aux grenouilles, sont recensées la Leptodactylus labrosus ainsi que, par$\mathrm{mi}$ les espèces occupant la forêt sèche équatoriale, les grenouilles Colostethus elachyhistus, Epipedobates anthonyi, Eleutherodactylus lymani (Pristimantis lymani), Leptodactylus labrosus et Physalaemus sp. (ibid:14). La présence d'une espèce dans une zone donnée n'est cependant pas immuable. Des changements ont pu survenir depuis l'époque mochica et d'autres espèces entrer en contact avec cette société par le biais de corridors naturels, de réseaux d'échanges, etc. Des restes appartenant à la grenouille de Junín, Batrachophrynus macrostomus, espèce d'altitude des Andes centrales, ont ainsi été découverts sur le site mochica de la Huaca de la Luna (Cárdenas, Rodríguez et Aguirre : 147). Les interactions qui en découlent ont, dès lors, pu influer sur la perception et les représentations de l'animal.

\section{Une iconographie combinatoire empreinte de symbolisme environnemental}

Les choix pictographiques exprimés dans les figurations d'amphibiens anoures montrent toute une gamme de déclinaisons où de nombreux éléments placent l'animal sur le chemin d'une fertilité naturelle et métaphysique oscillant entre réalisme et syncrétisme.

10. On peut trouver, par exemple, l'amphibien anoure identifié comme une tortue (Lehman, planche 62) ou encore comme un spécimen hybridé avec un singe (Collection du British Museum de Londres, $\mathrm{N}^{\circ}$ d'inventaire Am.B52.3).

11. L'amphibien anoure est tour à tour désigné sous le nom de grenouille (Longhena et Alva : 106 ; Maurer et Hennen : 200; Golte : 178) ou de crapaud (Franco et Vilela : 59; Ojalvo et Branca), selon la place que les auteurs attribuent à ces animaux dans le contexte andin. 


\section{Une figuration animale diversifiée}

L'analyse des éléments du corpus nous a amené à formuler plusieurs observations quant à la représentation des amphibiens anoures par les artisans mochicas. L'animal est figuré de différentes façons: seul ou en groupe, inclus dans des scènes, orné de détails corporels plus ou moins nombreux, matérialisé sous des traits diversement stylisés, etc. En règle générale, il se discerne de manière suffisamment explicite.

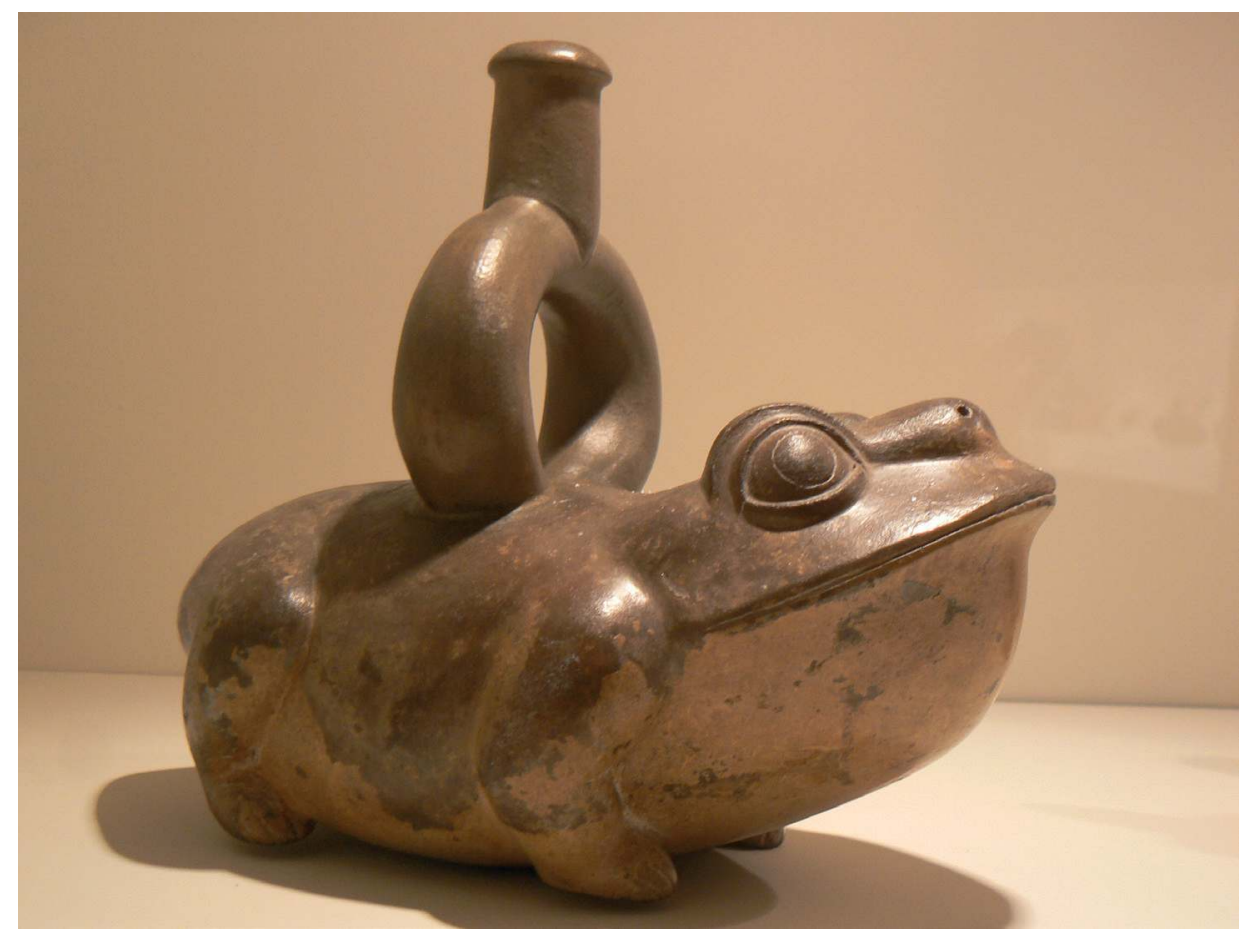

Figure 3 - Bouteille à anse goulot étrier représentant un amphibien anoure. Musée de la Nation, Lima, Pérou (Photo auteur).

Sa tête possède de nombreuses spécificités et variantes anatomiques: yeux, bouche et nez sont majoritairement présents, sous des déclinaisons diverses. Les membres antérieurs et postérieurs, lorsqu'ils peuvent être identifiés, sont principalement fléchis et munis de doigts. Quelques cas montrent un postérieur allongé ou un début d'appendice sur l'arrière train, pouvant correspondre à la configuration que prend le corps lorsque les membres postérieurs sont repliés près du tronc. La représentation en ronde-bosse est, par ailleurs, largement prédominante au regard des animaux en haut-relief (ou appliques), en bas-reliefs ou figurés en peinture. Le corpus de bouteilles céramiques permet également de déceler plusieurs critères iconographiques 
typiques de certaines phases chrono-culturelles ainsi que des évolutions de traitement, notamment en ce qui concerne l'emplacement de l'animal sur l'objet.

Outre les nombreux décors géométriques accompagnant les anoures - ouverts pour une grande part à la subjectivité interprétative -, ces animaux apparaissent en association avec d'autres éléments sélectionnés dans l'environnement naturel péruvien. Les interactions entre espèces peuvent, alors, renvoyer à une cohabitation constatée dans la nature, ou exprimer un lien symbolique entre les acteurs. Environ un tiers du corpus comporte des végétaux - sauvages ou agricoles - dont le type et la fréquence d'apparition sur l'anoure montrent un accroissement de la diversité botanique figurée à partir de la phase Mochica IV. Les fleurs interviennent de manière récurrente, sous des formes parfois très stylisées. S'il s'avère délicat de déterminer les espèces via l'iconographie, il semble néanmoins pertinent de voir dans bon nombre de représentations la matérialisation de la fleur de lotus (Nymphaea $s p$.). Parmi la faune, les reptiles sont figurés, majoritairement, sous la forme de serpents, prenant parfois l'aspect d'un animal bicéphale ou à tête de félin. Or, un parallèle s'observe entre le comportement du serpent et celui de l'anoure, tous deux animaux cycliques réapparaissant au début de la saison humide (Bourget 1994 : 144) et associés, en ce sens, au culte de l'eau ainsi qu'à la résurgence saisonnière depuis le monde souterrain ${ }^{12}$. Grues et échassiers composent, par ailleurs, la majorité des espèces d'oiseaux rencontrées, disposés au sein de décors peints où l'anoure est généralement le seul élément en relief. Au milieu de l'abondance de la faune et de la flore des eaux douces, la grue commence sa période de reproduction à la saison sèche (Golte : 170), complétant ainsi aux côtés de l'amphibien le cycle naturel et symbolique de la vie. Trois représentations murales - dites Thèmes Complexes - (Franco et Vilela), conservées sur les édifices archéologiques de deux sites mochicas (la Huaca de la Luna, à environ $5 \mathrm{~km}$ au sud-est de la ville de Trujillo dans la vallée de Moche, et la Huaca Cao Viejo, située dans la vallée de Chicama et à une cinquantaine de kilomètres au nord-ouest de Trujillo) témoignent également de cette inclusion de l'anoure dans un plus vaste ensemble iconographique, celui-ci véhiculant visuellement de nombreux éléments de la culture symbolique, religieuse et rituelle mochica.

12. La réapparition, au moment des pluies, de ces espèces jusqu'alors tapies, a pu leur conférer un symbolisme en lien avec leurs comportements communs et leur caractère propitiatoire. Les céramiques représentant ces deux espèces les placent, généralement, dans une position de cohabitation ou de confrontation (Giuliato : 137), observable dans la nature (Schulte). 


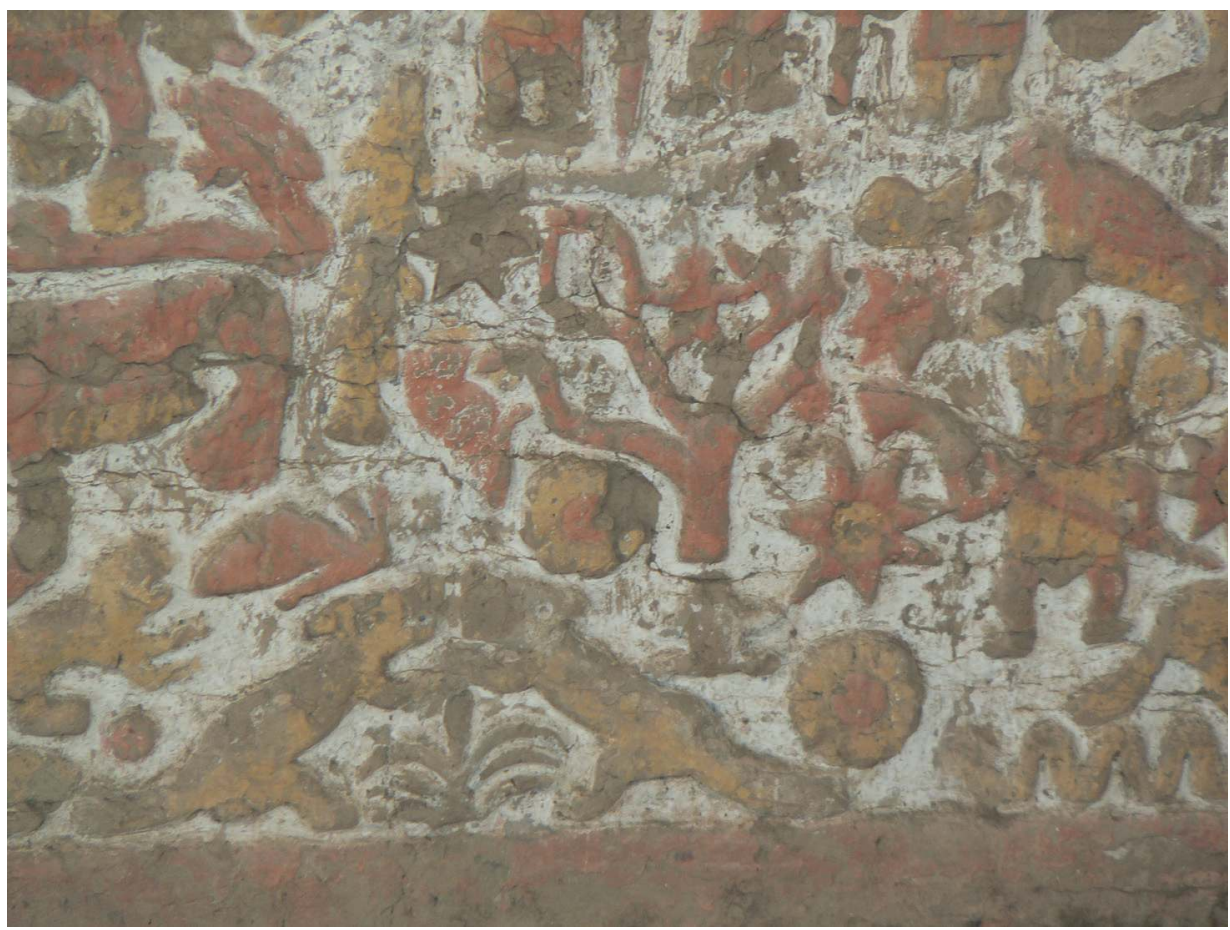

Figure 4 - Détail de la peinture murale dite Thème Complexe 1. Il présente, au sein d'un abondant décor, un amphibien anoure placé sous un arbre et entouré d'un félin ainsi que de plusieurs éléments géométriques en forme de fleur ou d'étoile. Huaca de la Luna, Pérou (Photo auteur).

\section{L'appel des pluies et la fonction reproductrice}

La fertilité semble être une notion associée naturellement par les peuples à l'amphibien anoure (Maurer et Hennen; Kalnicka : 179-182; Mazière : 141). Dans un environnement aride tel que celui occupé par les Mochicas, cette idée paraît particulièrement à-propos. La fonction régénératrice de l'animal, audelà de la notion stricto sensu de fécondité, est amplifiée par son rôle d'intermédiaire capable de favoriser une certaine renaissance de la terre ${ }^{13}$. Les nombreuses représentations sur lesquelles l'anoure à la tête inclinée en arrière, comme guettant les cieux, nous semblent parler en faveur d'une invocation des pluies. L'implication physique et active de l'amphibien engendrerait une action

13. En Australie, des témoignages aborigènes associent les amphibiens anoures à l'eau et à la saison des pluies, et par extension en font le symbole du renouveau de la vie suite à l'épanouissement de la faune et de la flore qui en découle (Boll et McArthur : 50). Quant au rôle d'intermédiaire dévolu à l'animal, il est perceptible au travers de croyances, notamment celle rapportée dans les Andes selon laquelle il « faut battre [les grenouilles] pour obtenir des ondées » (Mozzani : 828). 
directe - et remarquée par les Mochicas puisque figurée - dans le contrôle de l'eau. Par ailleurs, un renflement de la gorge d'amplitude variable se rencontre dans $25 \%$ des figurations, et ce au cours de toutes les phases culturelles (Giuliato : 125). Dans la nature, les anoures mâles attirent les femelles par des chants produits grâce à des sacs vocaux - situés dans la gorge, sur les flancs du cou ou sur les côtés de la tête qui se remplissent d'air lors de l'émission du son (O’Shea et Halliday: 17). Le gonflement porté sur ces représentations pourrait être la traduction du caractère sonore et par extension, reproducteur, de l'animal. En revanche, l'abondance qui caractérise le dépôt des œufs et l'éparpillement des petits ne paraît pas avoir été sujet à figuration. Seuls des décors circulaires placés sur le dos de l'animal pourraient symboliser les œufs portés, dans la nature, par certains individus adultes ou, métaphoriquement, les œufs expulsés lors de la ponte. Les larves et têtards n'ont, a priori, pas plus fait l'objet de représentations spécifiques. A environnement côtier équivalent, la culture Nasca (Silverman et Proulx) - qui s'est développée au sud du Pérou à une époque contemporaine de la culture Mochica, avant de disparaître au viI ${ }^{\mathrm{e}}$ siècle apr. J.-C. - a clairement mis en parallèle les différents stades d'évolution de ces animaux (Proulx : 158-161). D’après nos observations, les sociétés de la côte nord, en revanche, ne semblent pas avoir choisi ce trait biologique comme symboliquement significatif.

La culture Mochica a, toutefois, impliqué les anoures dans des scènes d'accouplement axillaire, position où le mâle grimpe sur le dos de la femelle et l'agrippe au niveau des membres antérieurs. Cependant, ces représentations céramiques montrent des animaux de taille sensiblement équivalente, alors que dans la nature le mâle est, sauf exception, d'une taille inférieure à celle de la femelle. D'autre part, la présence occasionnelle d'un partenaire d'une autre espèce, s'accouplant avec un amphibien anoure, constitue un second critère en faveur d'une fonction non naturaliste de ces représentations. La valeur de l'acte résiderait, alors, dans le symbolisme de l'association animale et non dans une stricte mimèsis. L'idée de fertilité, ainsi véhiculée, relie sans doute les amphibiens anoures aux rituels " destinés à générer la reproduction de la société [qui] s’obtenait par le biais des activités agricoles " (Hoquenghem, cité par Fraresso : 81).

\section{Un « animal sauvage agricole »}

Le lien entre la venue des pluies et la croissance agricole apparait comme primordial au travers des productions iconographiques mochicas. L'amphibien anoure, au demeurant animal sauvage, se voit rattaché aux éléments propres à l'agriculture. Cette représentation d'un anoure botanique, sorte d'animal sauvage agricole, est entourée d'une forte codification. En effet, lorsque des végétaux sont matérialisés sur le corps du batracien, ils se conforment à un type de disposition 
particulier ; ainsi certaines espèces sélectionnées sont placées sur les flancs alors que d'autres sont localisées sur le postérieur de l'anoure.

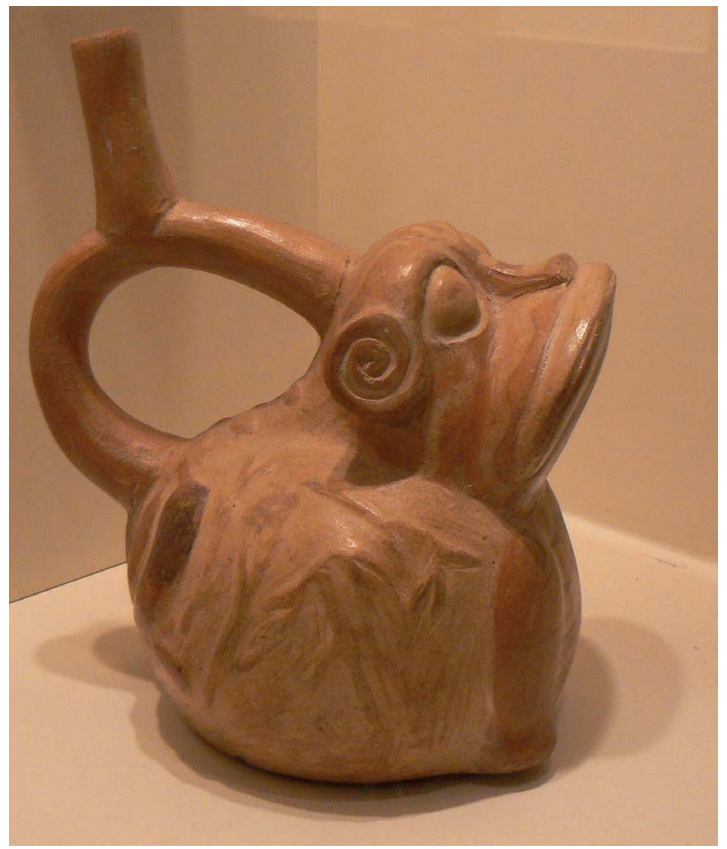

Figure 5 - Bouteille à anse goulot étrier représentant un amphibien anoure botanique. Son flanc droit est orné de plantes de maïs, son dos et son arrièretrain présentent respectivement la tige et les tubercules de manioc. Musée Larco, Lima, Pérou. N d'inventaire ML007171 (Photo auteur).

L'espèce la plus fréquemment rencontrée est le manioc, ou $y u c c a$, parfois identifié comme du piment ${ }^{14}$; nous soutenons, pour notre part, l'affirmation de Donna McClelland privilégiant les tubercules de manioc (McClelland: 31), du fait de l'association récurrente de décors en losange sur le dos de l'animal - motifs ressemblant de façon très nette aux excroissances présentes sur la tige de la plante. De plus, son fréquent positionnement sur l'arrière-train rappelle les œufs que certains anoures portent sur cette partie de leur anatomie, en période de reproduction. La fertilité de l'amphibien serait alors directement corrélée à la multiplication et à la croissance symbolique du tubercule. Parmi les autres espèces cultivées, le pallar apparaît sur 47 pièces du corpus, majoritairement sur le corps de l'anoure, liant l'animal à cette espèce de haricot (Phaseolus sp.) qui nécessite une courte période d'arrosage et peut générer jusqu'à quatre récoltes annuelles (Golte : 209).

\section{L'anoure félin botanique : un syncrétisme hérité et particularisé}

Une certaine mixité entre amphibiens anoures et félins s'observe également dans les productions mochicas. Ce thème, mêlant les détails anatomiques des deux espèces, a sans doute une origine antérieure, héritée de la culture Chavín-Cupis-

14. Pour exemple, nous renvoyons vers une des pièces de la collection du Musée Larco à Lima, $\mathrm{n}^{\circ}$ d'inventaire ML007136, dont la description dans le catalogue en ligne (http:// www.museolarco.org/catalogo/) indique un crapaud, ou une grenouille, ayant du maïs et des piments sur le corps. 
nique ( $\mathrm{X}^{\mathrm{e}}-\mathrm{II}^{\mathrm{e}}$ siècle av. J.-C. env. $)^{15}$. Des anoures comportant des oreilles, et/ou des crocs, et/ou des griffes se rencontrent, ainsi, dans $15 \%$ du corpus (Giuliato : 149).

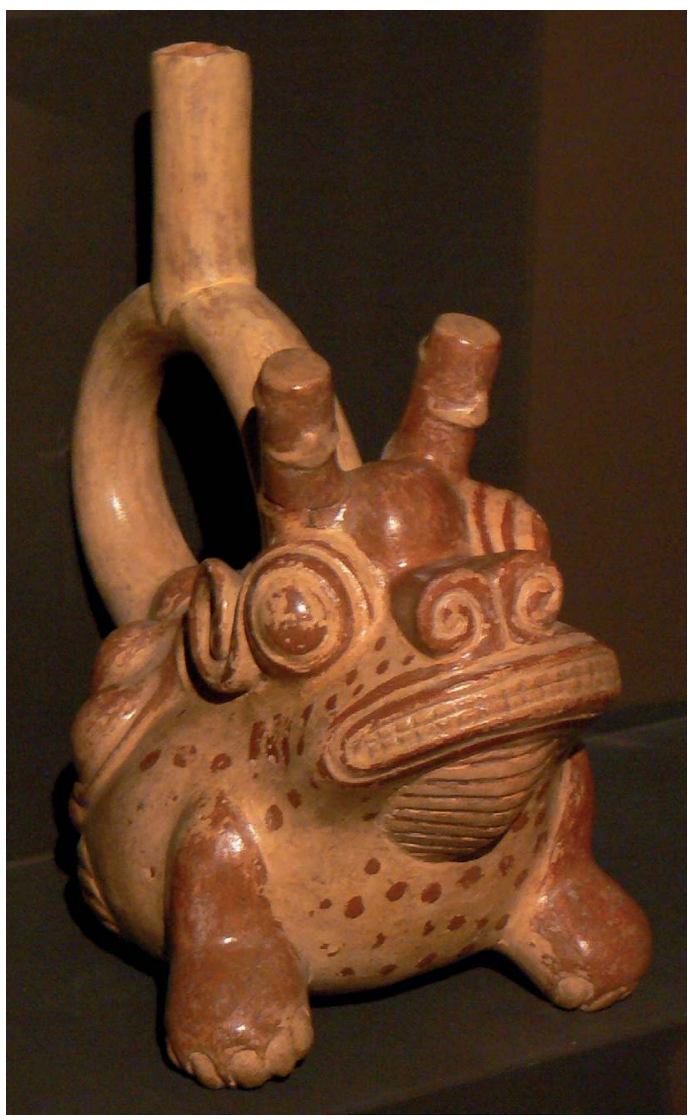

Figure 6 - Bouteille à anse goulot étrier représentant un anoure félin botanique. L'animal est pourvu de griffes, de dents, d'une bosse frontale et d'un nez spiralé, ainsi que d'un large décor en spirale dans le prolongement de l'œil. Son arrière-train comporte des tubercules de manioc et deux tiges de la plante émergent de sa tête. Musée Archéologique National Brüning, Lambayeque, Pérou. $N^{\circ}$ d'inventaire MB00546 (Photo auteur).

Or, des données ethnologiques ont permis de mettre en relation le comportement des pumas et la réapparition des amphibiens à la saison des pluies (De Bock : 162). Le phénomène d'El Niño amplifie d'autant cette association (Ibid. 162), les fortes pluies favorisant le retour de ces animaux dans la sphère du visible et à proximité immédiate des hommes. Notons que le félin est la seule autre espèce avec laquelle l'anoure s'accouple dans les scènes d'amplexus ${ }^{16} ;$ la présence systématique de végétaux - sur l'amphibien ou sur le félin - semble attribuer à l'acte une valeur symbolique de croissance et de fécondité végétale. L'accouplement serait une déclinaison - peut-être légèrement plus tardive - de la figure préexistante de l'anoure félin botanique ${ }^{17}$, venant expliquer la genèse de l'animal hybride ou

15. À ce sujet, voir notamment la synthèse de Rebecca Stone-Miller sur l'art des cultures andines.

16. Nom donné à la technique d'accouplement de la plupart des amphibiens anoures, se déclinant en amplexus lombaire ou axillaire, selon que le mâle s'agrippe à la femelle au niveau des membres postérieurs ou antérieurs.

17. L'archéologue Rafael Larco Hoyle a identifié, dans ses travaux iconographiques, l'enfantement d'une créature hybride : le crapaud-félin végétal, issu de l'accouplement d'un crapaud et d'un félin dont les flans sont ornés de végétaux ou de fruits (Larco 
en approfondir le sens métaphysique. Cette association résulterait, selon A.M. Hocquenghem (1977: 8), d'un accouplement mythique entre le crapaud et le jaguar, qui serait en relation avec une légende sur l'origine des récoltes abondantes. Cet animal peut faire l'objet d'une représentation complexe, combinant éléments anatomiques caractéristiques (dents, griffes, nez orné d'une double spirale, larges spirales en relief dans le prolongement de l'œil et pouvant évoquer des oreilles, gorge légèrement enflée) avec la présence de tubercules de manioc sur le postérieur et de tiges de manioc sur le sommet du crâne (Giuliato : 151). Qu'il s'agisse de mêler les spécificités supposées des deux espèces animales (monde terrestre/monde souterrain) ou d'établir un lien direct avec l'abondance végétale, cette mise en scène exprime les variations stylistiques et métaphoriques d'un thème propre aux Mochicas. Son apparition sur les bouteilles en céramique à partir de la phase Mochica III pourrait correspondre à une adaptation de la pensée symbolique comme conséquence d'une transformation induite par des phénomènes sociaux ou climatiques (ibid.).

Le thème de la croissance agricole, émanant du corps de l'animal, semble également migrer, dans les phases tardives, vers une iconographie à la dimension fonctionnelle accrue, qu'elle soit d'ordre utilitaire ou symbolique $^{18}$. Une scène peinte, ainsi que plusieurs autres représentations en basrelief de phase Mochica IV (Ibid.: 154), témoignent d'une stylisation au thème identique mais selon des formes encore renouvelées; de la même façon, les phases Tardive et Transitionnelle de la zone Mochica Nord présentent, sur des cántaros, les attributs de l'animal hybride sous des traits réinterprétés (Ibid. : 99).

Hoyle: 105). Anne-Marie Hocquenghem cite également la présence, dans l'iconographie mochica, d'un « crapaud mythique » du corps duquel poussaient des plantes cultivées (Hocquenghem : 157). Par la suite, Donna McClelland a étendu les recherches au sujet de ce "Botanical Frog» (ou "grenouille botanique ") en apportant des données concernant les plantes qui lui sont associées, les critères félins de l'amphibien et sa présence dans des scènes de procession peintes (Donna McClelland: 37). Pour notre part, considérant que les critères de détermination spécifique sont insuffisants pour avoir recours au terme de grenouille ou de crapaud, nous employons l'expression " anoure félin botanique ", synthétisant les trois critères qui le caractérisent : appartenance aux amphibiens anoures, éléments félins et présence de végétaux.

18. À titre d'exemple, une céramique de période Transitionnelle du site de San José de Moro (tombe M-U615) illustre, ainsi, l'évolution iconographique de l'anoure botanique dans les phases tardives mochicas : les végétaux qui ornaient le corps y ont perdu de leur part symbolique en cédant la place à la figuration d'objets et d'ustensiles dont la valeur semble plus pragmatique et en lien avec les activités humaines (agricoles et rituelles) (Giuliato : 161). 


\section{Un médiateur qui transcende les mondes}

Doté de qualités amphibies, l'anoure semble acquérir, dans la pensée mochica, un rôle d'intermédiaire capable d'accompagner l'individu et de se mouvoir au travers des différentes sphères du monde visible et invisible.

\section{L'amphibien anoure entre eau, terre et ciel}

Le positionnement sur l'objet, en fonction notamment de certaines zones colorées, tend à mettre en évidence l'existence de frontières au bord - ou au travers - desquelles l'animal se place. Ce symbolisme de délimitations spatiales, qu'elles soient visibles ou symboliques, amène à considérer les capacités amphibies propres à l'anoure chez lequel l'aptitude à transcender les univers se trouve renforcée par diverses associations iconographiques.

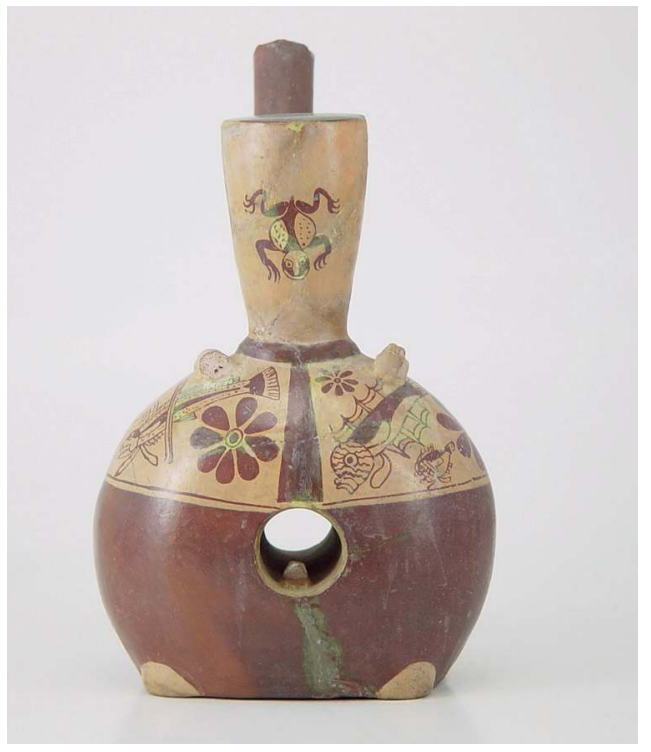

Figure 7 - Bouteille à anse goulot étrier comportant un amphibien anoure peint le long du col. Deux autres anoures sont placés en relief sur une panse décorée de fleurs de lotus, de poissons et d'oiseaux. Un dernier batracien est présent à l'intérieur de l'orifice circulaire réalisé dans la partie monochrome de la panse. Musée Larco, Lima, Pérou. Nº d'inventaire ML006754 (Photo Musée Larco).

Une céramique ${ }^{19}$ met le batracien ${ }^{20}$ en relation avec le crustacé dit marucha, dont la présence sur les plages littorales péruviennes lui confère un statut d'intermédiaire entre le monde aquatique et le monde terrestre (Golte : 177), à l'image de la fleur de lotus représentée de manière récurrente auprès des anoures. Le pélican brun du Pérou accompagne également l'amphibien ; vivant en permanence sur la côte, cet oiseau a la particularité de courir sur quelques mètres à la surface de l'eau pour pouvoir prendre son envol ${ }^{21}$, parvenant ainsi, comme l'anoure, à se maintenir à la limite entre

19. Collection du Musée de la Nation, Lima (Golte : 177).

20. Le terme batracien est communément employé pour désigner grenouilles et crapauds. 21. La rubrique «Pélican» du dictionnaire Larousse en ligne (http://www.larousse.fr/ encyclopedie/vie-sauvage/p\%C3\%A9lican/178178, consulté le 5 juillet 2015) décrit, dans un paragraphe sur le pélican brun d'Amérique (Pelecanus occidentalis), ce phénomène lié au fouettement de l'eau par les pattes de l'animal et qui lui permet de prendre son essor avant de partir en vol. 
deux mondes. Médiateur capable d' «appeler » les pluies par sa simple présence ou par ses capacités sonores, nous remarquons, en outre, que l'amphibien est très largement représenté orienté vers le ciel, de par sa localisation sur l'objet ou son inclinaison corporelle (Giuliato : 128). La recrudescence des chants d'anoures avant les épisodes pluvieux amplifie son rôle d'intercesseur ; l'animal pouvait, alors, entrer dans des rites de conciliation de la nature, en qualité de messager capable de porter prières et offrandes auprès des divinités. Au Pérou, le processus conduisant à reconnaître ce pouvoir à l'anoure se rencontre au travers de pratiques communautaires décrites notamment au siècle dernier (Hocquenghem : 252). Différents rites visaient, ainsi, à attirer les pluies nécessaires à la croissance agricole et à la vie, comme celui consistant à faire "pleurer $»^{22}$ un amphibien déposé dans une céramique jusqu’à ce que son message ait été véhiculé (Vellard 1991 : 462). Le haricot, lui-même - présent sur 47 pièces de notre corpus (Giuliato : 146) et évoqué précédemment pour son rendement agricole - revêt une symbolique d'intercesseur dans la culture Mochica (Golte : 217), et sa présence sur les céramiques à l'effigie des anoures renforce d'autant cet aspect de lien entre monde terrestre et monde souterrain.

\section{Entre monde des morts et des vivants}

La majorité des céramiques de notre étude provenant de contextes funéraires, il convient de les replacer dans le cas particulier du dépôt d'offrandes. L'amphibien anoure accompagnait, ainsi, symboliquement, le défunt dans l'après-vie, la mort constituant un passage vers l'inframonde et, éventuellement, le monde des ancêtres mythiques (Bourget 1990 : 59). La société mochica a fréquemment eu recours à des dépôts d'animaux, de diverses espèces, au sein des sépultures humaines (Goepfert). Pour autant, à notre connaissance, aucun amphibien anoure n'a été l'objet de ce type d'offrande dans une tombe mochica (Giuliato : 70 et 175). Ceci contraste singulièrement avec la quantité de céramiques représentant des anoures qui ont été déposées lors d'inhumations.

Peut-être l'image matérielle suffisait, alors, à générer sa présence, la valeur étant transférée à l'objet façonné qui se retrouvait doté des capacités et symboles que l'on imputait à l'animal de son vivant.

Plusieurs autres aspects postulent en faveur d'un rôle actif des anoures dans les croyances liées à la mort. Des exemples céramiques de notre corpus illustrent, ainsi, le thème de l'enterrement, où l'animal occupe une place

22. Ce verbe est mis en avant par Jean Vellard dans la citation où il rapporte une scène caractéristique se déroulant sur l'Île du Soleil (Lac Titicaca) en période de sécheresse. L'action de "pleurer» est générée par l'abandon de grenouille(s) dans des céramiques disposées au sommet d'une montagne ; les amphibiens se mettent alors à " appeler » la pluie, qui vient les délivrer en remplissant d'eau les céramiques (Vellard : 462). 
particulière, seul élément en relief au sein d'une scène complexe (Ibid: 162). Un amphibien modelé se trouve, par ailleurs, placé à l'intérieur d'un conduit peint par lequel le défunt rejoint la tombe et l'autre monde, renforçant l'idée d'un accès à un autre plan dimensionnel (Ibid. : 163).

Cette perspective transparaît, également, dans l'organisation de l'espace funéraire ; à l'intérieur d'une sépulture du site de Ventarrón ${ }^{23}$, une céramique en forme d'anoure a été découverte disposée aux pieds du défunt, comme en lien avec la terre et le monde souterrain (Ibid. : 103-104). Notons que les Mochicas n'envisageaient pas la mort comme une fin mais comme une rupture, une perturbation de la vie qui venait inverser le processus terrestre et au cours de laquelle le défunt accédait à un nouvel espace. Un rapprochement se constate, aussi, au travers des "rituels liés à l'ancestralité [qui] devaient avoir lieu aux alentours du mois de novembre et se terminaient lors de la «sortie des morts ", c'est-à-dire au début de la saison des pluies " (Hocquenghem, cité par Fraresso : 97). Nous retrouvons, ici, l'aptitude à l'hibernation du Bufo lors de la saison sèche, lequel peut soudainement réapparaître en surgissant de terre à la période des pluies. Cette résurrection symbolique rencontre un écho supplémentaire dans l'association récurrente de l'animal avec les tubercules de manioc, les deux espèces manifestant une même tolérance à l'enfouissement prolongé (Nye, cité par McClelland : 35). Le positionnement de l'amphibien anoure autour du trou de certaines panses ${ }^{24}$ céramiques rajoute à ce symbolisme du passage d'un environnement (ouvert/fermé, intérieur/extérieur) à un autre, illustrant par là cette " capacité à transgresser périodiquement les milieux» (Goepfert : 511), naturels ou métaphysiques. Par ailleurs, la présence de l'animal sur un couteau cérémoniel (Giuliato : 109) le relie aux actions sacrificielles au cours desquelles le sang des hommes aurait fait office d'offrande miroir pour l'eau des montagnes, dont on espérait l'arrivée dans les fleuves côtiers (Bourget 1998 : 46). Pour autant, ce rôle de médiateur que nous lui prêtons n'est pas réservé à l'amphibien anoure. Les multiples espèces animales déposées dans les sépultures mochicas indiquent que nombre d'entre elles étaient investies - sous des formes et à des degrés divers - d'une fonction d'intermédiaire entre "sphères terrestres, célestes et chtoniennes » (Goepfert : 445). Dans ce cadre, l'anoure occupe, cependant, une place spécifique, qui nous apparaît comme un auxiliaire sollicitant le maintien des cycles naturels et facilitant le nécessaire cheminement vers le renouveau.

23. Tombe 14, site archéologique de Ventarrón, vallée de Lambayeque.

24. La panse est la partie renflée de la céramique. 


\section{Les Mochicas et les amphibiens anoures : entre assimilation et dissociation}

Les témoignages matériels semblent parler en faveur d'une extension de la valeur du batracien dans les pratiques sociales du peuple Mochica, à la fois par l'utilisation des substances biologiques de l'animal à des fins rituelles et par le choix qui peut s'être opéré, parmi les espèces d'anoures, de celles propres à être figurées.

\section{S'adjoindre les propriétés de l'animal}

Les capacités d'accompagnement de l'anoure se trouvent amplifiées par certaines implications thérapeutiques et symboliques, qui s'observent notamment dans des décors zoomorphes réalisés sur des céramiques en forme de portraits qui suggèrent l'hypothèse de possibles tatouages corporels (Giuliato : 135). Plusieurs exemples présentent, ainsi, un amphibien peint sur le menton ou les joues d'un visage humain, souvent identifié comme un chaman.

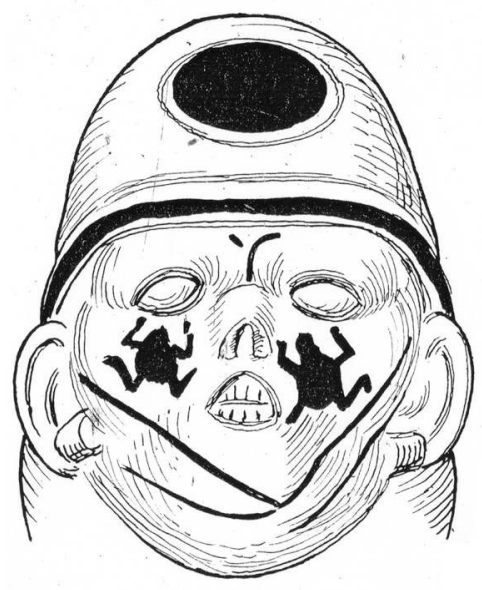

Figure 8 - Dessin du détail d'une bouteille à anse goulot étrier comportant un personnage avec deux amphibiens anoures peints sur les joues. Les lèvres et le cartilage du nez de l'individu montrent des signes de déformation pathologique (Wassen 1934 : 353-354, fig. 25).

Le personnage pourrait transporter ou mastiquer de la coca (Donnan, cité par Goepfert, : 94) afin de favoriser les visions et entrer en contact avec le monde supérieur et celui des morts. Il serait, alors, aidé par l'anoure, peutêtre en qualité de médiateur vers le monde souterrain ou de par d'éventuelles propriétés d'altération neurologique - les batraciens étant dotés de diverses glandes sécrétant, généralement, du mucus ainsi que du venin à la toxi-

cité variable (MacQuarrie : 257). Parmi les animaux disposés conjointement à l'anoure se rencontrent serpent, poissons life ${ }^{25}$ et mammifères carnivores (renards ou pumas ?). Ils pourraient, ensemble, participer à un processus de transformation symbolique inter-espèces par lequel l'homme effectuerait une métamorphose totale ou partielle (Magni : 177), ou se déplacerait mentalement entre monde terrestre et aquatique. Les appendices plus ou moins marqués, présents sur certains amphibiens figurés, renverraient, alors, au vestige de queue disparaissant lors du passage à l'âge adulte, mettant ainsi l'accent sur la mutation biologique de

25. Signalons que des tatouages de ce poisson d'eau douce ont été découverts sur les avant-bras de la Dame de Cao, sur le site mochica de la Huaca Cao Viejo (Mujica, cité par Gálvez Mora et Runcio : 66). 
l'animal dont l'existence aquatique devient terrestre après acquisition des pattes et du système pulmonaire.

D'autre part, les anoures entrent dans la composition de divers remèdes à travers le monde (Vellard : 462 ; Collingham : 48), les animaux étant employés morts ou vivants, selon les coutumes et le résultat désiré. La région septentrionale du Pérou conserve de nombreuses croyances les concernant, certaines plus spécifiquement en relation avec les crapauds (Frisancho Pineda ; Giuliato : 192-193). Il semble que des pratiques aient toujours cours, notamment dans la région de Túcume où, en 2009, il nous a été rapporté qu'un des « sorciers ", alors en exercice, employait un récipient en pierre en forme de batracien.

Par ailleurs, dans $55 \%$ des cas, les représentations mochicas d'amphibiens anoures possèdent un décor à l'arrière de l'œil (Ibid.: 121). La mise en avant de cette partie du corps, la constance de son occurrence et la variété des formes qu'elle adopte, dénotent, selon nous, l'importance de cet élément anatomique, peut-être en lien avec les glandes parotoïdes et les propriétés de leurs toxines ${ }^{26}$.

Un fruit nommé ulluchu (Guarea sp.), bien qu'absent du littoral désertique péruvien, s'observe sur plusieurs céramiques, ainsi que sur le couteau en métal de notre corpus. La composition chimique de cette plante, appuyée par les représentations iconographiques, indique qu'elle était employée en contexte sacrificiel pour, notamment, augmenter la pression sanguine (Bussmann et Sharon : 5). Les graines, préparées et inhalées, ont également pu avoir un effet psychotrope, causant une altération des capacités mentales, ainsi que de rapides mais courtes hallucinations ( Ibid.).

De plus, à l'instar des poisons contenus dans le corps des amphibiens, les tubercules de manioc qui leur sont si souvent associés sont pareillement toxiques mais peuvent, dans certains cas, être consommés après transformation (McClelland : 35). Bien que le sujet ne fasse pas consensus (Giuliato : 22-23), il semble que l'utilisation des sécrétions du Bufo marinus soit envisageable et transposable dans les rites mochicas. Car, si l'action hallucinogène de la bufoténine, présente dans les toxines des crapauds, fait toujours débat (Weil et Davis : 4), certaines sources admettent que la substance peut être isolée et utilisée, comme ce fut le cas sur divers continents (Polia : 29 ; Ratsch).

\section{Des espèces aux formes distinctes et aux symboles évolutifs}

Il convient, enfin, de considérer plusieurs facteurs découlant de la coexistence des espèces et de la variabilité des systèmes symboliques.

26. Situées à l'arrière des yeux, les glandes parotoïdes font partie de l'appareil vénéneux des crapauds (O'Shea et Halliday : 224). Les toxines des crapauds du genre Bufo d'Amérique du Sud comportent diverses caractéristiques et propriétés, leurs substances se rencontrant de façon variable en fonction des espèces (Giuliato : 22). 
Se nourrissant d'une grande quantité d'insectes nuisibles aux cultures, le crapaud est souvent considéré comme un animal bénéfique pour les agriculteurs. Pourtant, certains comportements, événements climatiques ou pratiques rituelles ont pu lui adjoindre une facette plus négative, qui n’a pas forcément été partagée par toutes les espèces d'anoures. Car les amphibiens n'assument pas nécessairement les mêmes rôles sociaux; ainsi au Pérou, toutes les grenouilles ne sont pas en mesure d' " appeler» la pluie (Granadino et Jara Jimenez : 33). Au vu des circonstances changeantes auxquelles est soumis le littoral péruvien, il est possible d'envisager que l'amphibien anoure ait été paré d'une certaine dualité : une face bénéfique associée à l'eau et à la fécondité, une autre destructrice à l'œuvre lors de violentes intempéries et inondations. L'accroissement d'épisodes extrêmes a pu, dans certaines vallées et à certaines dates, amener à considérer l'anoure comme un animal néfaste, annonçant - voire incitant - les catastrophes climatiques liées aux pluies, aux sécheresses et aux séismes. Par ailleurs, un caractère hostile a pu être attribué à une espèce en particulier, de par son impact sur les activités humaines ou environnementales. À ce propos, soulignons, par exemple, l’ambivalence exprimée par les Dhalwangu d'Australie qui, bien que protecteurs des grenouilles, voient dans le crapaud Bufo marinus un animal malveillant (Boll et McArthur : 49).

Notre hypothèse selon laquelle le peuple Mochica n'aurait pas accordé une symbolique uniforme à toutes les espèces d'amphibiens anoures se trouve renforcée par les physionomies prises par les animaux figurés. Procéder à la détermination spécifique des espèces au travers des vestiges matériels permettrait de mieux appréhender les relations particulières existant entre l'homme et l'animal. Toutefois, cette identification est rendue malaisée en raison du manque de détails et du degré interprétatif variable des représentations, n’autorisant pas la transposition d'un protocole scientifique basé sur des données herpétologiques précises.

Cependant, d'autres critères visuels et biologiques peuvent se révéler pertinents et conduire à diverses propositions. Ainsi, de par la comparaison symbolique avec les comportements des félins et l'occupation des zones agraires, les crapauds correspondraient plus volontiers à l'image de l'anoure félin et/ou botanique - plusieurs combinaisons étant rencontrées, alors que les grenouilles, au mode de vie nettement amphibie, pourraient avoir été privilégiées dans les scènes naturalistes où elles apparaissent parmi la faune et la flore côtières (Giuliato : 148).

Ceci étant, même si nous pensons que les espèces de grenouilles et crapauds ont fait l'objet de représentations distinctes, les limites du rapprochement iconologie/ biosphère s'établissent précisément dans le vaste éventail des possibilités offertes. Nos observations concernant la figuration des anoures témoignent toutefois de disparités spatiales - en particulier entre les zones Mochica Nord et Sud - et temporelles. À l'instar de l'iconographie mochica, le thème subit des évolutions 
de traitement- perceptibles notamment via l'anoure félin botanique, le thème de l'enterrement ou encore l'association croissante d'objets - sans doute influencées par les aléas climatiques. Ainsi les Thèmes Complexes de la Huaca Cao Viejo (site archéologique d'El Brujo) auraient été réalisés à une époque tardive, en réaction à un passage particulièrement désastreux d'El Niño (Shimada, cité par Franco et Vilela : 126), à des fins de restauration de l'ordre naturel. Plusieurs épisodes arides précis ont également été datés (Dillehay et Kolata), notamment entre 563 et 594 apr. J.-C., époque correspondant à la transition entre les phases Mochica Moyen et Mochica Tardif (Shimada, cité par Hill : 529). Les modes de représentations des anoures suggèrent, eux aussi, cette mutation du rôle de l'animal au sein d'une iconographie rituelle tentant de s'adapter aux périodes de crise, dans le but de rétablir et de maintenir l'équilibre sociétal (Franco et Vilela : 126).

\section{Conclusion}

Si l'on considère les représentations d'amphibiens anoures comme des mises en forme de la pensée sociale mochica, alors les créations matérielles nous permettent de percevoir la place particulière occupée par ces animaux au sein d'une vaste conception métaphysique de la nature. Les points évoqués soulignent le symbolisme complexe des anoures, dénotant une empreinte marquée et à multiples particularismes, qui prend sens également dans le cadre culturel élargi de l'aire andine (Quilter, cité par Fraresso : 12).

Si l'on considère la préexistence du thème de l'anoure félin, alors il apparaît que la culture Mochica l'a adopté et l'a fait perdurer avec un succès indéniable tout au long de ses phases chrono-culturelles. L'ajout de l'élément végétal est une innovation mochica qui semble éclore à une époque précise et, à l'image de l'anoure félin, ne rencontrer qu'une postérité limitée au travers de rares exemples au sein des cultures Sicán $\left(\mathrm{VIII}^{\mathrm{e}}{ }^{\mathrm{e}} \mathrm{XIV}^{\mathrm{e}}\right.$ siècle apr. J.-C.) et Chimú ( $\mathrm{XII}^{\mathrm{e}}$ $\mathrm{xv}^{\mathrm{e}}$ siècle apr. J.-C) (Giuliato : 201-211). Les scènes d'amplexus, figurées par de nombreuses cultures péruviennes et américaines, bénéficient, ici, d'un traitement normalisé qui applique des critères de figuration codifiés d'où transparaît une forte valeur symbolique (ibid. : 155-158). Nous avons pu, par ailleurs, apprécier la complexité des tentatives de détermination spécifique, où les critères d'identification biologique restent, pour une grande part, inapplicables à une source iconographique (ibid. : 179-183).

Pourtant, il nous parait évident que crapauds et grenouilles ont fait l'objet de productions différenciées, dont la perception présenterait un réel intérêt ; tout comme un accroissement des données en matière de datation et de localisation permettrait une compréhension plus fine de l'évolution de la pensée du peuple mochica et de sa relation spécifique et prolifique avec les espèces d'anoures. 


\section{Bibliographie}

BOLL Valérie M., McARTHUR Lorrae. "Sur les traces de Garkman, la grenouille, dans le Nord-Est de la Terre d'Arnhem (Australie) », pp. 41-54. Journal de la Société des Océanistes, $\mathrm{n}^{\circ}$ 120-121 (2005).

BOURGET Steve. «Des tubercules pour la mort. Analyses préliminaires des relations entre l'ordre naturel et l'ordre culturel dans l'iconographie mochica", pp. 45-85. Bulletin de l'Institut Français d'Etudes Andines, vol. 19, nº 1 (1990).

BOURGET Steve. Bestiaire sacré et flore magique : écologie rituelle de l'iconographie de la culture mochica, côte nord du Pérou. Ph. D. dissertation, Département d'Anthropologie, Université de Montréal, 1994 (2 tomes).

BOURGET Steve. " Pratiques sacrificielles au site Moche de la Huaca de la Luna ", pp. 41-74. Bulletin de l'Institut Français d'Etudes Andines, vol. 27, n 1 (1998).

BOURGET Steve, JONES Kimberly (sous la dir. de). The Art and Archaeology of the Moche, An Ancient Society of the Peruvian North Coast. Austin, University of Texas Press, 2008, 315 p.

BUSSMANN Rainer W., SHARON Douglas. "Naming a Phantom - the Quest to Find the Identity of Ulluchu, an Unidentified Ceremonial Plant of the MocheCulture in Northern Peru", pp. 1-6. Journal of Ethnobiology and Ethnomedecine, vol. $5, \mathrm{n}^{\circ} 8$ (2009).

CAPULA Massimo. Les batraciens et les reptiles. Paris, Éditions Solar, coll. « Guide Vert », 1990, 256 p.

CARDENAS J., RODRIGUEZ J., AGUIRRE L. «El material orgánico en Huaca de la Luna ", p. 128-149. Investigaciones en la Huaca de la Luna 1995. S. UCEDA, E. MUJICA, R. MORALES (sous la dir. de). Trujillo, Proyecto Arqueológico Huacas del Sol y de la Luna-Facultad de Ciencias Sociales-Universidad Nacional de La Libertad, 1997.

CASTILLO BUTTERS Luis Jaime et al. Arqueología mochica : nuevos enfoques. Actas del Primer Congreso Internacional de Jóvenes Investigadores de la Cultura Mochica, Lima 4 y 5 de agosto de 2004. Lima, Fondo Editorial de la Pontificia Universidad Católica del Perú, 2008.

COLLINGHAM Lizzie. Le curry, une histoire gastronomique de l'Inde. Paris, Éditions Petite Bibliothèque Payot, 2009.

DE BOCK Edward K. «Pumas gordos y la época de las lluvias : sacrificio, transición y status real en la iconografía Moche y Chimú », p. 159-168. Desarrollo arqueológico costa norte del Perú. Tome 2. Luis VALLE ALVAREZ (sous la dir. de). Trujillo, Ediciones Sian, 2004, 406 p.

DESCOLA Philippe. "L'envers du visible : ontologie et iconologie». Histoire de l'art et anthropologie. Paris, Institut National d'Histoire de l'Art/Musée du quai Branly, 2009, mis en ligne le 28 juillet 2009. URL : http://actesbranly.revues. org/181, consulté le 5 juillet 2015 . 
DILLEHAY Tom D., KOLATA Alan L. "Long-term human response to uncertain environmental conditions in the Andes", pp. 4325-4330. Proceedings of the National Academy of Sciences of the United States of America, vol. 101, $\mathrm{n}^{\circ} 12$ (2004).

FRANCO JORDAN Régulo, VILELA PUELLES Juan. El Brujo, el mundo mágico religioso mochica y el calendario ceremonial. Trujillo, Ediciones Minka Perú, 2005, $141 \mathrm{p}$.

FRARESSO Carole. L'usage du métal dans la parure et les rites de la culture Mochica (150-850 apr. J.-C.), Pérou. Thèse de doctorat. Université Michel de Montaigne-Bordeaux, 2007.

FRISANCHO PINEDA David. Curanderismo y brujería en la costa peruana. Lima : s.n., 1986, 93 p.

GALVEZ MORA César A., RUNCIO María Andrea. «El life (Trichomycterus sp.) y su importancia en la iconografía mochica", p. 55-87. Archaeobios, vol. 1, $\mathrm{n}^{\circ} 3$ (2009).

GIULIATO Sandra. Archéologie d'une socialisation de la nature dans l'aire andine précolombienne : l'empreinte des amphibiens anoures dans la culture Mochica (ier-Ixe siècle. apr. J.-C.), Pérou. Mémoire de Master 2 Recherche. Université Michel de Montaigne-Bordeaux 3, 2012 (non publié).

GOEPFERT Nicolas. Rites funéraires, offrandes et sacrifice animal dans l'idéologie religieuse de la culture Mochica (100-800 apr. J.-C), côte nord du Pérou. Thèse de doctorat. Université Paris I Panthéon-Sorbonne, 2009.

GOLTE Jürgen. Moche - Cosmología y Sociedad, una interpretación iconográfica. Cuzco, Instituto de Estudios Peruanos-Centro de Estudios Regionales Andinos Bartolomé de Las Casas, 2009.

GRANADINO Cecilia, JARA JIMENEZ Cronwell. Las ranas embajadoras de la lluvia y otros relatos. Cuatro aproximaciones a la Isla de Taquile. Lima, Minka, 1996, $240 \mathrm{p}$.

HILL Erica. "Death as a Rite of Passage : The Iconography of the Moche Burial Theme", pp. 528-538. Antiquity, $\mathrm{n}^{\circ} 72$ (1998).

HOCQUENGHEM Anne-Marie. "Les érotiques" et l'iconographie mochica », pp. 6-14. Objets et Mondes, vol. 17, nº 1 (1977).

HOCQUENGHEM Anne-Marie. Iconografía Mochica. Lima, Fondo Editorial de la Pontificia Universidad Católica del Perú, 1989.

KALNICKA Zdenka. "Images of water and woman in the arts", pp. 170-190. Wagadu, 3 (2006).

KARADIMAS Dimitri. «Yurupari ou les figures du diable », pp. 45-58. Gradhiva, ${ }^{\circ} 6$ (2007).

KOON Michele, ALEX Bridget. "Revised Moche Chronology Based on Bavesian Models of Reliable Radiocarbon Dates", pp. 1039-1055. Radiocarbon, vol. 56, $\mathrm{n}^{\circ} 3$ (2014). 
LARCO HOYLE Rafael. Checan, essai sur les représentations érotiques du Pérou précolombien. Genève, Éditions Nagel, 1965, 146 p.

LAVALLEE Danielle. Les représentations animales dans la céramique mochica. Paris, Musée de l'Homme-Mémoire de l'Institut d'Ethnologie, 1970, 320 p.

LEHMAN Walter. The Art of Old Peru. New York, Hacker Art Book, 1975, $128 \mathrm{p}$.

LONGHENA Maria, ALVA Walter. Les Incas. Les civilisations andines, des origines aux Incas. Paris, Gründ, 1999, 290 p.

MacQUARRIE Tim. Donde los Andes encuentran al Amazonas, BahuajaSonene y Madidi, Parques nacionales de Perú y Bolivia. Barcelona, Editorial Jordi Blassi, 2001.

MAGNI Caterina. "Coyolxauhqui et le were-jaguar: de l'objet d'art au mythe ", pp. 171-180. Cahiers des Amériques Latines, nº 25 (1997).

MAURER Evan, HENNEN Molly. Symboles sacrés, 4000 ans d'art des Amériques. Paris, Éditions de la Réunion des Musées Nationaux, 2002, 215 p.

MAZIERE Marlène. Art rupestre amérindien en Guyane française. Matoury, Ibis Rouge Éditions, 2008, 165 p.

McCLELLAND Donna [1989]. «The Moche Botanical Frog », pp. 30-42. Arqueología Iberoamericana, $\mathrm{n}^{\circ} 10$ (2011).

MILLAIRE Jean-François. "Primary state formation in the Virú valley, North Coast of Peru", pp. 6186-6191. Proceedings of the National Academy of Sciences of the United States of America, vol. 107, $\mathrm{n}^{\circ} 14$ (2010).

MOZZANI Eloïse. Le livre des superstitions - Mythes, croyances et légendes. Paris, Éditions Robert Laffont, 1995, 1822 p.

O'SHEA Mark, HALLIDAY Tim. Reptiles et amphibiens. 400 espèces. Paris, Éditions Bordas, 2001, 256 p.

OJALVO Catherine, BRANCA Marzia et al. Pérou, l'art de Chavín aux Incas. (Petit Palais - Musée des Beaux-Arts de la ville de Paris, 5 avril-2 juillet 2006). Milan, Éditions Skira, 2006, 223 p.

PARSONS Harry. The nature of frogs. Vancouver, Éditions Douglas \& McIntyre, 2000, $112 \mathrm{p}$.

PILLSBURY Joanne. Moche Art and Archaeology in Ancient Peru. Washington, Yale University Press, coll. "Studies in the History of Art Series", n 63, 2002, $344 \mathrm{p}$.

POLIA Mario. Il Perú prima degli Incas. Milan, Éditions Xenia Tascabili, 1998, $126 \mathrm{p}$.

PROULX Donald. A sourcebook of Nasca Ceramic Iconography. Iowa, University of Iowa Press, 2006, $274 \mathrm{p}$.

QUILTER Jeffrey. "Moche Politics, Religion, and Warfare", pp. 145-195. Journal of World Prehistory, vol. 16, $\mathrm{n}^{\circ} 2$ (2002). 
RATSCH Christian. The Encyclopedia of Psychoactive Plants: Ethnopharmacology and its Applications. Rochester, Park Street Press, 2004, 944 p.

SANTIANI Marc. Amphibiens et reptiles. Saint-Armand-Montrond, Éditions Artémis, 2002, $128 \mathrm{p}$.

SCHULTE Rainer. «Bufo glaberrimus (Gúnther 1868) un raro batracio de la cordillera oriental del departamento de San Martín », p. 61-64. Boletín de Lima, $\mathrm{n}^{\circ} 73$ (1991).

SILVERMAN Helaine, PROULX, Donald. The Nasca. Oxford, John Wiley \& Sons Ltd, coll. "Peoples of America Serie", 2002, 360 p.

STONE-MILLER Rebecca. L’art des Andes : de Chavín aux Incas. Paris, Éditions Thames \& Hudson, 1996, 224 p.

VELLARD Jean. "Los Batracios", pp. 453-462. El Lago Titicaca: síntesis del conocimiento limnológico actual. Claude DEJOUX, André ILTIS (sous la dir. de). La Paz, Institut Français de Recherche Scientifique pour le Développement en Coopération/Office de la Recherche Scientifique et Technique Outre-Mer - Instituto de Historia Social Boliviana, 1991.

VENEGAS Pablo J. « Herpetofauna del bosque seco ecuatorial del Perú : taxonomía, ecología y biogeografía », p. 9-26. Zonas Aridas, vol. 9 (2005).

WEIL Andrew, DAVIS Wade. «Bufo alvarius : a Potent Hallucinogen of Animal Origin », pp. 1-8. Journal of Ethnopharmacology, vol. 41, n 1-2 (1994).

Résumé : La société mochica ( $\mathrm{I}^{\mathrm{er}}-\mathrm{Ix} \mathrm{e}$ s. apr. J.-C.) du Pérou a produit de nombreux témoignages à l'effigie des amphibiens anoures. L'étude de ces représentations révèle des modes de figuration variés, parfois codifiés, évoluant dans le temps et l'espace. Les éléments significatifs choisis par les Mochicas pour intégrer la matérialisation de l'animal permettent, dans la limite des hypothèses autorisées, de prendre la mesure de la riche et singulière place occupée par le thème au sein de cette culture. Les différents genres et espèces, peuplant notamment la côte septentrionale du Pérou, semblent avoir été investis d'un rôle dont nous présentons ici quelques facettes, évoquant, entre autres, l'emprise du monde agricole, le recours au syncrétisme animalier et l'anoure en tant que médiateur.

Mots clés : Pérou, précolombien, Mochica, grenouilles, crapauds.

Abstract: Moche society, in pre-Columbian Peru, created many objects reproducing the shape of anuran amphibians. The study of these representations shows a variety of figurative modes, in some cases codified, that evolved through time and places. The significant features selected by the Moche to portray the animal allow us, within the limits of the study's hypotheses, to measure the rich and singular specificities of this theme in Moche material culture. The multiple genera and species, in particular those living on the north coast of Peru, seem to play a multi-faceted role related to the impact of agricultural life, animal syncretism and the mediating role of the anuran, amongst other themes.

Keywords: Peru, pre-Columbian, Moche, frogs, toads

Biographie : Sandra Giuliato est historienne de l'art et guide-conférencier. Ses recherches portent sur les liens entre cultures précolombiennes du Pérou et amphibiens anoures au travers des vestiges archéologiques. Également diplômée en ethnologie, elle s'intéresse aux rites et croyances des peuples du continent américain, et de l'arc antillais, en relation avec ces animaux et l'écosystème 
en général. Elle a participé à plusieurs fouilles archéologiques programmées et s'implique dans la médiation en qualité de présidente de l'association Archéanthrope.

Biography: Sandra Giuliato is an art historian and guide. Her research focuses on the relation between pre-Columbian cultures and anuran amphibians through archaeological evidence. She also holds a degree in social anthropology and is interested in the rites, symbolisms and popular beliefs found in the cultures of the American continent and Antillean islands about these specific animals as well as the larger ecosystem. She has volunteered on several archaeological digs and is involved in mediation work as part of her term as president for the non-profit organization Archeanthrope. 